\title{
Probable Nipa Palm Wine-Associated Hepatitis A Outbreak after Attending a Funeral Ceremony in Sabah
}

\author{
Muhammad Jikal, ${ }^{1}$ Daisuke Mori, ${ }^{2}$ Ahmad Faudzi Yusoff, ${ }^{3}$ Sarawasthi Bina Rai, ${ }^{4}$ M. Hafiz Mukhsam, ${ }^{1}$ Ismail Ali, ${ }^{1}$ Liza Abd Latif, ${ }^{5}$ \\ Alfred A. S. Perianayagam, ${ }^{6}$ Esther Bernad, ${ }^{1}$ Cynthia C. E. Bailon, ${ }^{5}$ Lim Guan Lee, ${ }^{5}$ Vielerian Fredolin, ${ }^{6}$ and Kamruddin Ahmed ${ }^{2,7 *}$ \\ ${ }^{1}$ Sabah State Health Department, Ministry of Health, Kota Kinabalu, Sabah, Malaysia; ${ }^{2}$ Department of Pathobiology and Medical Diagnostics, Faculty \\ of Medicine and Health Sciences, Universiti Malaysia Sabah, Kota Kinabalu, Sabah, Malaysia; ${ }^{3}$ Institute of Medical Research, Ministry of Health, Jalan \\ Pahang, Kuala Lumpur, Wilayah Persekutuan Kuala Lumpur, Malaysia; ${ }^{4}$ Aimst University, Bedong, Kedah, Malaysia; ${ }^{5}$ Beaufort Health Office, Ministry \\ of Health, Pekan Beaufort, Beaufort, Sabah, Malaysia; ${ }^{6}$ Papar Health Office, Ministry of Health, Bandar Papar, Papar, Sabah, Malaysia; ${ }^{7}$ Borneo \\ Medical and Health Research Centre, Faculty of Medicine and Health Sciences, Universiti Malaysia Sabah, Kota Kinabalu, Sabah, Malaysia
}

Abstract. Foodborne outbreaks of hepatitis A virus (HAV) are most commonly associated with fresh and frozen produce and with various types of shellfish. Alcoholic beverage-borne outbreaks of hepatitis A are extremely rare. Here, we report an outbreak of hepatitis $A$ associated with the consumption of a traditional wine at a funeral ceremony in the Sabah state of Malaysian Borneo. Confirmed cases were determined by serum anti-HAV immunoglobulin M and/or for fecal HAV by reverse transcription polymerase chain reaction (RT-PCR). The amplicons of RT-PCR were subjected to nucleotide sequencing followed by phylogenetic analysis. We conducted a 1:2 case-control study to identify the possible exposure that led to the outbreak. Sixteen patients met the case definition, they were 18 to 58 years old and $90 \%$ of them were males. The case-control study showed that the consumption of nipa palm wine during the ceremony was significantly associated $(P=0.0017)$ with hepatitis A infection (odds ratio, 5.44; $95 \% \mathrm{Cl}, 1.80-16.43$ ). Untreated river water was used to dilute the traditional wine, which was assumed to be the source of the infection. Phylogenetically, these viruses belonged to genotype IA and formed an independent cluster with strains from Taiwan, Japan, and the Philippines. This strain might be an emerging HAV in Asian countries. Environmental assessments were performed and environmental samples were negative for HAV. The incidence of hepatitis A in Sabah was also determined and it was 0.795/100,000 population. Strict monitoring of traditional wine production should be implemented by the local authority to prevent future outbreaks.

\section{INTRODUCTION}

Every year, an estimated 1.5 million people are infected by hepatitis A virus (HAV) worldwide, ${ }^{1}$ which is most likely an underestimation because of the asymptomatic presentation of, and limited epidemiological information on this disease. ${ }^{2}$ The population prevalence of HAV infection depends on several socioeconomic factors, such as the density of housing, sanitation, quality of water, and income. Overall improvements of such conditions worldwide are leading to a shift in the susceptibility to infection from an early age to young and even older ages. ${ }^{2}$ Traditionally, the endemicity of HAV is classified into low, intermediate, and high levels based on the prevalence of anti-HAV immunoglobulin G (lgG) in the human serum, which reflect a seropositivity of $<15 \%, 15-50 \%$, and $>50 \%$, respectively. ${ }^{2}$ Malaysia is a low endemic country for $\mathrm{HAV}^{3}$ where $100 \%$ and $96 \%$ of the populations have access to at least basic sanitation services and drinking-water sources. ${ }^{4}$ The main surveillance system in Malaysia is the mandatory notification of infectious diseases under the Prevention and Control of Infectious Diseases Act 1988. Therefore, since 1988, hepatitis A is a notifiable disease in this country. Results from seroepidemiology studies showed that the national incidence rate had dropped steadily from $2.24 / 100,000$ population in 2000 to $0.41 / 100,000$ in $2013 .^{3}$ However, the health indices are not homogeneous throughout the country, and the prevalence of infectious diseases varies among states. Sabah is the second largest state in Malaysia, located at the northern portion of the Borneo Island. The state is consisting of 26 districts, with an estimated population of 3.9 million. Sabah has a relatively low

*Address correspondence to Kamruddin Ahmed, Department of Pathobiology and Medical Diagnostics, Faculty of Medicine and Health Sciences, Universiti Malaysia Sabah, 88400 Kota Kinabalu, Sabah, Malaysia. E-mail: ahmed@ums.edu.my urban population compared with other Malaysian states, where only $54 \%$ of Sabah's population live in urban areas. ${ }^{5}$ Healthcare facilities in Malaysia is mainly provided by the government, in Sabah there are 24 hospitals, 25 health offices, 134 health clinics, 30 community health clinics, 166 rural health clinics, 400 private clinics, and eight private hospitals.

Since 2006, cases of hepatitis A have been reported regularly in Sabah. However, little is known about the outbreaks, the genotype distribution of the circulating HAV and their relationship with other areas. Therefore, the present study was performed during an outbreak of hepatitis $A$ that occurred in Beaufort district of Sabah in December 2017. The objectives of this outbreak investigation were to describe its epidemiological characteristics, identify its source, determine the genotype of the outbreak strain, assess its relatedness with strains from other areas, identify the size of the problem, and contain the outbreak.

\section{MATERIALS AND METHODS}

Incidence rate of hepatitis A. The yearly numbers of notified hepatitis A cases were extracted from the database of the Sabah State Health Department and analyzed. To calculate

TABLE 1

Demographic features of cases and controls

\begin{tabular}{lcc}
\hline & Cases $(N=16)$ & Controls $(N=32)$ \\
\hline Age & 8 & \\
$15-30$ years old & 6 & 18 \\
$31-50$ years old & 2 & 6 \\
$\quad>50$ year old & 15 & \\
Gender & 1 & 14 \\
$\quad$ Male & & 18 \\
Female & 16 & 32 \\
Citizenship & 0 & 0 \\
$\quad$ Malaysian & 0 \\
\hline
\end{tabular}


80

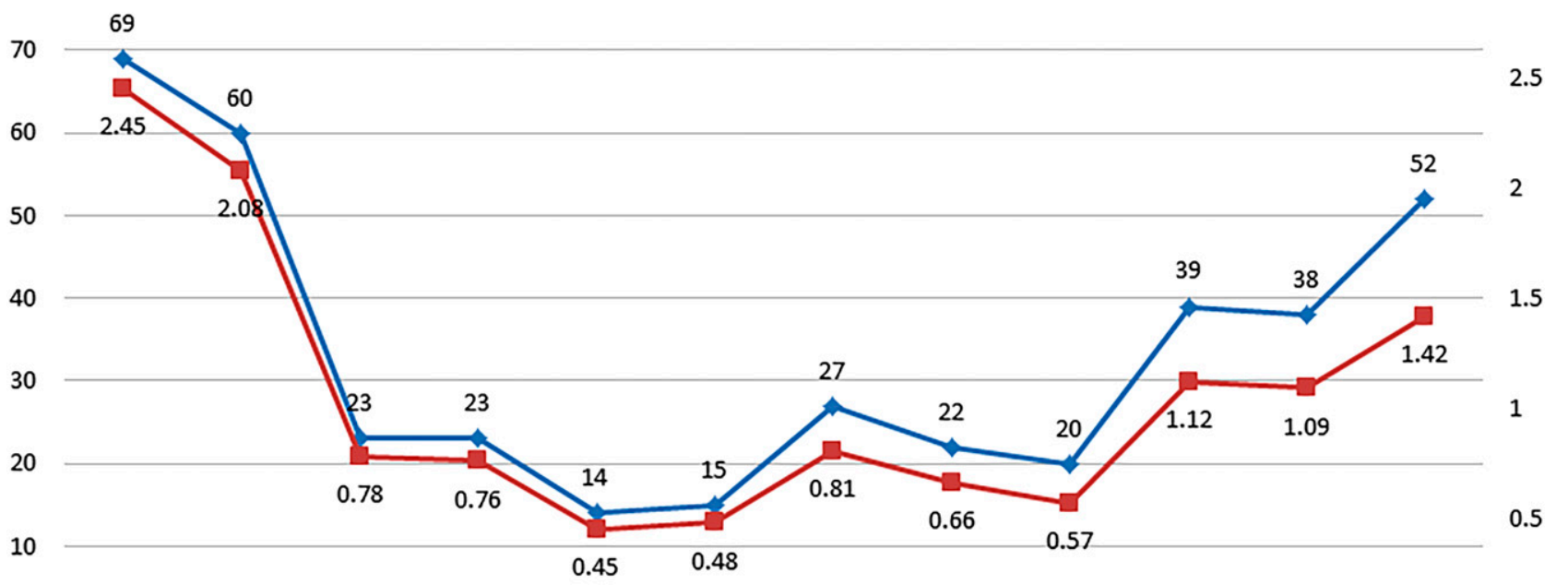

0

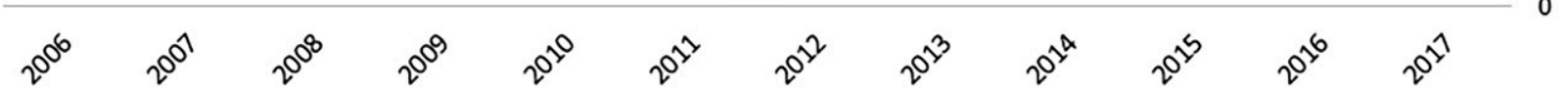

$\neg$ Number of cases $\quad-$ - Incidence rate

FIGURE 1. The yearly number of notified hepatitis A cases (blue line) in Sabah and their incidence rate (orange line) during 2006-2017. The incidence rate is presented per 100,000 populations.

the incidence rate, the number of notified HAV cases during the 2006-2017 period were divided by the population of Sabah state for each matching year. The incidence rate was calculated per 100,000 populations.
Chronology of the events. On December 15, 2017, the Sabah State Health Department started an outbreak investigation after receiving notifications of 16 suspected cases of hepatitis A from the Beaufort District Health Office between

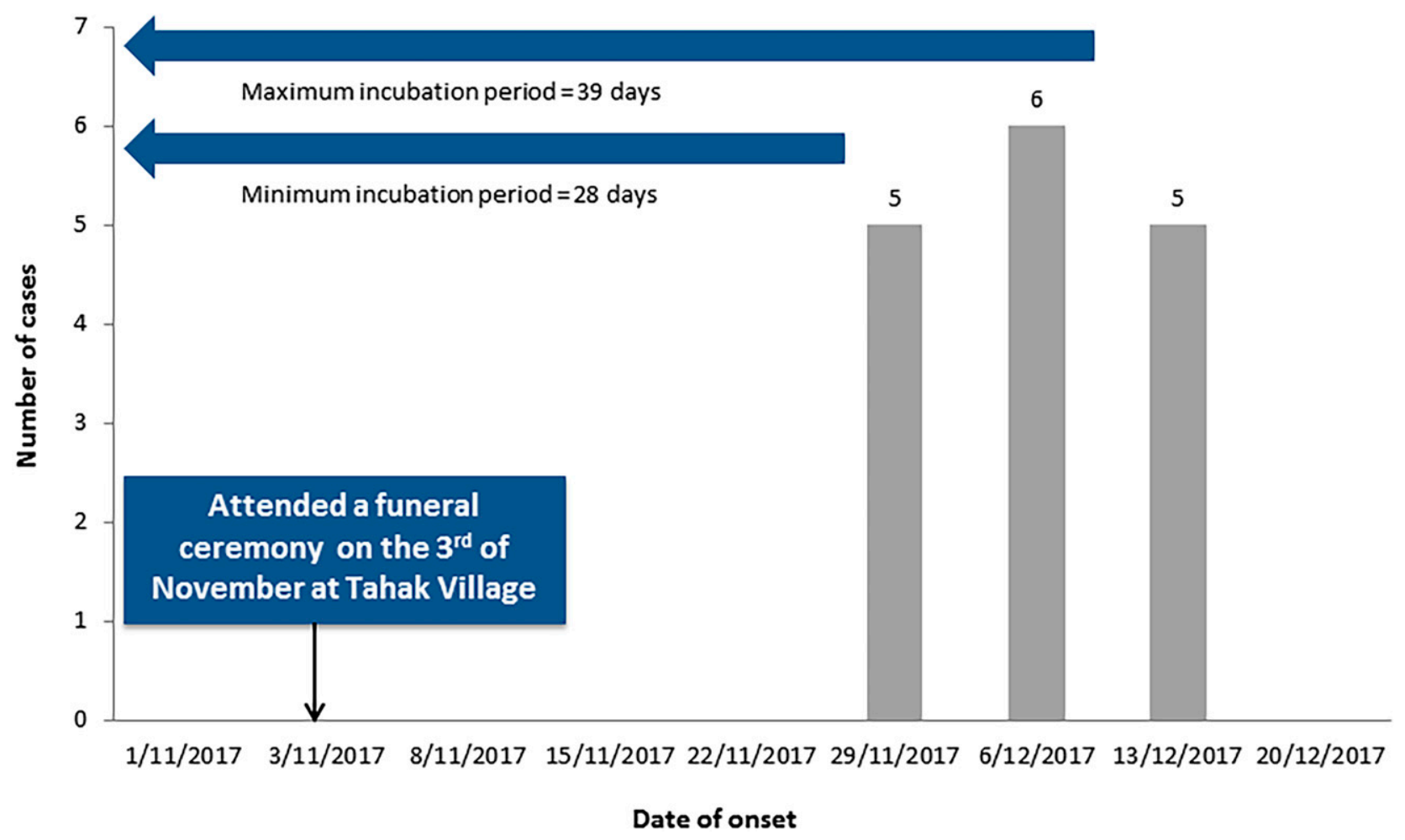

FIGURE 2. The epidemic curve of the hepatitis A outbreak in Tahak village, Beaufort District, Sabah, Malaysia. The number of cases was plotted against the day of the occurrence of fever and jaundice. 
December 8 and 15, 2017. These patients were admitted in different hospitals. Since hepatitis $A$ is a notifiable disease therefore, these cases were identified by notification from different hospitals. Fever and jaundice were the main clinical presentations, accompanied by elevated liver enzymes. It was found that all patients had attended a funeral ceremony in Tahak village in Beaufort District. Tahak village is a traditional village with a population of about 600 people that has proper sanitation and water supply. Its main economic activity is agriculture. All cases were Malaysian from the Kadazan ethnicity. To rule out leptospirosis, a microagglutination test (MAT) was performed and that yielded negative results in 15 patients. By this time, one of the patients tested positive for the immunoglobulin M antibody against HAV (anti-HAV IgM) in the serum.

Cases were defined as those who had attended the funeral service at Tahak village, Beaufort District, on November 3, 2017, and who presented with fever and jaundice with or without nausea in the period afterward. Confirmed cases were those who were either positive for serum anti-HAV IgM and/ or for fecal HAV by reverse transcription polymerase chain reaction (RT-PCR). The identification of active cases was conducted via a house-to-house survey using a structured questionnaire $^{6}$ that included questions on sociodemographic parameters, history of attending the funeral service, and activities related to the possible ways of transmission. Similarly, 79 contacts were also investigated. For passive case finding, all Ministry of Health facilities in Beaufort district, and private general practitioners were alerted and requested to notify the Beaufort District Health Office of any cases of this illness.

A case-control study with a 1:2 ratio of cases and controls was also performed to determine whether consumption of a particular food during the funeral ceremony was associated with the outcome. The controls were selected among the attendees of the funeral ceremony who did not present with any symptoms. The demographic features are shown in Table 1. Environmental observation and assessment included the examination of water sources, sanitation, and waste management using the standard investigation form. Environmental samples that include clams, ice, and water used at the wet market, and vegetables and fruits collected from the market, were sent to the National Public Health Laboratory at Sungai Buloh, Selangor, Malaysia.

Laboratory analysis of serum and stool samples. Ten serum samples were sent to a private diagnostic laboratory in Kota Kinabalu, for the detection of anti-HAV IgM using an enzyme-linked immunosorbent assay (Abbott Laboratories, Chicago, IL), according to the manufacturer's instructions. Fifteen stool samples were sent to the Department of

TABLE 2

Distribution of Hepatitis A cases according to clinical features

\begin{tabular}{lc}
\hline Symptoms and signs & Number of cases (\%) \\
\hline Fever & $16(100)$ \\
Jaundice & $16(100)$ \\
Chills & $12(75)$ \\
Headache & $8(50)$ \\
Muscle pain & $4(25)$ \\
Abdominal pain & $3(19)$ \\
Nausea & $3(19)$ \\
Vomiting & $2(13)$ \\
Joint pain & $2(13)$ \\
Cough & $2(13)$ \\
\hline
\end{tabular}

Pathobiology and Medical Diagnostics, Faculty of Medicine and Health Sciences, Universiti Malaysia Sabah for HAV detection and genotyping by RT-PCR. Fecal suspensions (10\% suspension in phosphate-buffered saline [PBS] solution $[\mathrm{pH}$ 7.4]) were treated with chloroform, and aliquots were stored at $-80^{\circ} \mathrm{C}$ until extraction. RNA was extracted from stool samples using the QIAamp Viral RAN Mini Kit (Qiagen, Hilden, Germany) and subjected to cDNA synthesis using Superscript III First-Strand Kit (Invitrogen Corporation, Carlsbad, CA). Nested PCR for the VP1/2A region of the HAV genome was performed. ${ }^{7}$ The amplified products were separated by agarose gel electrophoresis and stained with ethidium bromide.

The nucleotide sequence of the amplicons was determined using the BigDye Terminator Cycle Sequencing Kit (v. 3.1; Applied Biosystems, Foster City, CA), according to the manufacturer's instructions, and the product was run on an ABI Prism 3100 Genetic Analyzer (Applied Biosystems). Partial nucleotide sequences of the VP1/2A were used for phylogenetic analyses. The nucleotide sequences of other HAVs were extracted from GenBank. Multiple sequence alignment was carried out using ClustalW, and a phylogenetic tree was constructed using MEGA 7.0 by applying the neighbor-joining method. Bootstrap analysis of 1,000 replicates was conducted to determine the significance of the branching of the constructed tree.

\section{RESULTS}

The yearly median number of cases of hepatitis A in Sabah is 25 (range, 14-69 cases; 95\% Cl, 21.92-45.08) cases. The median incidence rate of hepatitis $A$ is 0.795 (range, 0.45-2.45; 95\% Cl, 0.6519-1.4598) cases $/ 100,000$ population (Figure 1).

A total of 16 hepatitis A cases were reported in the four following villages: Mandangin (one case), Dungau (four cases), Tahak (nine cases), and Parang Besar (two cases). All of them were primary cases, none of these cases were detected by active surveillance. Our investigations revealed that all cases had attended a funeral ceremony at Tahak village on November 3, 2017. Nearly 150 people attended the ceremony, yielding an overall attack rate at of $10.6 \%$. The cases were aged between 18 and 58 years and $90 \%$ were male. The epidemiological curve indicated a common-source type of outbreak (Figure 2). The mean incubation period was 32 days. All 16 cases presented with fever and jaundice (100\%), chills in 12 cases $(75 \%)$, and other clinical features as shown in Table 2. There was no death and all cases recovered without any events. The case-control study revealed that the

TABLE 3

Univariate analysis of food taken during the funeral ceremony

\begin{tabular}{lll}
\hline \multicolumn{1}{c}{ Food items } & \multicolumn{1}{c}{ Odds ratio $(95 \% \mathrm{Cl})$} & $P$ value \\
\hline Chicken in soy sauce & $1.22(0.33-4.46)$ & 1.000 \\
Buffalo meat in soy sauce & $6.00(0.87-41.44)$ & 0.0235 \\
Mineral water & $1.56(0.50-4.86)$ & 0.692 \\
White rice & $1.71(0.45-6.54)$ & 0.6749 \\
Syrup water & $0.87(0.31-2.46)$ & 1.000 \\
Pork in sauce & $1.31(0.48-3.58)$ & 0.7055 \\
Rice wine & $2.25(0.84-6.05)$ & 0.2512 \\
Beer & $3.50(1.95-6.29)$ & 0.1034 \\
Nipa palm wine & $5.44(1.80-16.43)$ & 0.0017 \\
\hline
\end{tabular}




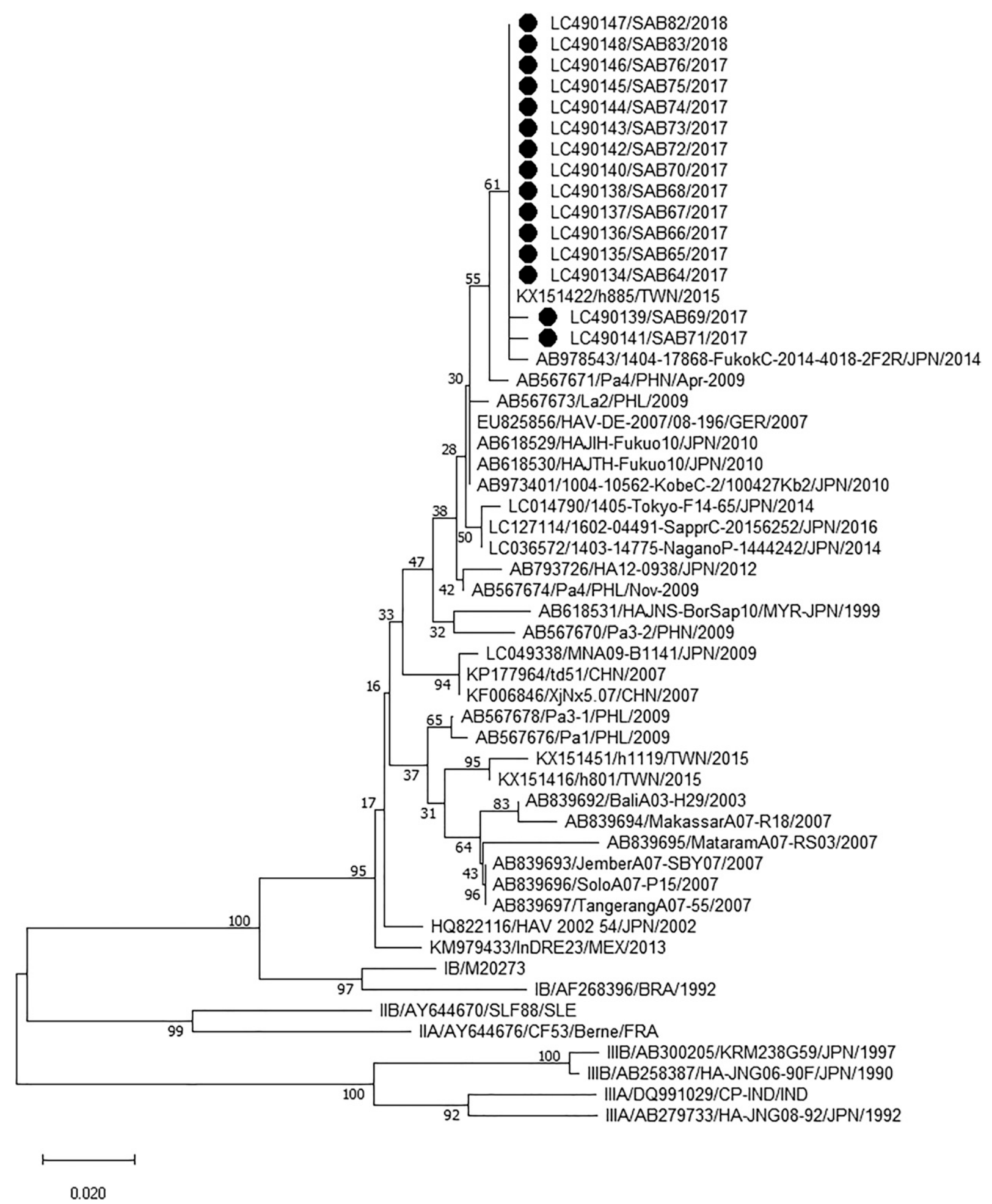

FIGURE 3. Phylogenetic tree constructed using the partial nucleotide sequences of the VP1/2A region of the hepatitis A virus genome via the neighbor-joining method. The strains analyzed in this study are marked with a filled circle. The number adjacent to the node represents the bootstraP value. The scale bar at the bottom indicates the genetic distance expressed as nucleotide substitutions per site. The nucleotide sequences of our strains have been submitted to the databases of the DNA DataBank of Japan, the European Molecular Biology Laboratory, and GenBank (accession nos. LC490134 to LC490148).

consumption of palm wine and buffalo meat in soy sauce during the ceremony were significantly associated $(P=0.0017$ and 0.035 , respectively) with hepatitis $A$ infection (odds ratio [OR] $5.44 ; 95 \% \mathrm{Cl}, 1.80-16.43$ and OR $6.00 ; 95 \% \mathrm{Cl}$, $0.87-41.44$, respectively) (Table 3 ).
A trace back investigation revealed that the wine was prepared traditionally. Sap from nipa palm (Nypa fruticans) plants was used as the source of carbohydrates. The individual who prepared the wine admitted that to increase the volume river water was mixed in during the preparation of the wine. It is 
also highly likely that the river water overflowed into the bottles during high tide. Observation of the wine preparation sites showed that the nipa palms were located at the bank of the river. Insects were found inside the sap-collecting containers, which were not clean (Supplemental Figure 1). There were illegal settlers near the sap-collection sites, where poor sanitation conditions were detected.

The serum or stool sample was unavailable in one case; therefore, it was diagnosed clinically. Ten serum samples were positive for anti-HAV IgM. All stool samples were positive for HAV by RT-PCR, and nucleotide sequencing of these amplicons and a subsequent Basic Local Alignment Search Tool confirmed the identity of the viruses as being HAV. The phylogenetic analysis revealed that the strains were of genotype IA (Figure 3). The cluster included to strains from Taiwan, Japan, and the Philippines. All environmental samples were negative for HAV by RT-PCR.

\section{DISCUSSION}

Hepatitis $A$ is rarely fatal ${ }^{1}$ and all cases analyzed here recovered without any adverse events. However, death occurs during outbreaks; ${ }^{8}$ therefore, outbreak of hepatitis A has a significant impact on public health in a low-prevalence country like Malaysia, and a place like Sabah, where the incidence rate of hepatitis $A$ is comparable to that of developed countries. ${ }^{9}$ Usually, hepatitis $A$ is associated with a lack of safe water, and poor sanitation and hygiene. ${ }^{2}$ Foodborne outbreaks of HAV have been associated most commonly with fresh and frozen produce, such as semidried tomatoes, frozen berries, and pomegranate arils, as well as with various types of shellfish, such as raw scallops and oysters, clams, and shrimps. ${ }^{10}$ However, alcoholic beverage-borne outbreaks of hepatitis $A$ are extremely rare. We found only two reports of this type of outbreak: one was reported in 2012 in the Perak state of Malaysia ${ }^{11}$ and the other occurred in India ${ }^{12}$ after consumption of a traditional wine called coconut toddy. This is an alcoholic beverage with an alcohol content of $4-5 \%$ made from the sap of coconut trees. The $\mathrm{pH}$ of coconut toddy ranges from 3.0 to 3.8 , at which HAV can survive and multiply. ${ }^{11}$ A study showed that drinking alcohol beverages containing $\geq 10 \%$ alcohol has a protective effect, whereas beverages containing $<10 \%$ alcohol has no or little effect on oysterborne hepatitis $A{ }^{13}$ Nipa palm wine has low alcohol content possibly that is the reason why people were not protected from hepatitis $A$.

A hepatitis $A$ outbreak associated with nipa palm wine has never been reported previously. Homemade unrecorded liquor production from different carbohydrate sources is widespread in Sabah. ${ }^{14}$ Our case-control study provided evidence that the consumption of nipa palm wine during the ceremony was significantly associated with hepatitis A infection. Although the buffalo meat in soy sauce showed a significant association with the infection, the $\mathrm{Cl}$ of this item is wider, indicating uncertainty associated with the estimate and it crosses 1, which implies the absence of difference from the control. ${ }^{15}$ Furthermore, buffalo meat in soy sauce is cooked well at high heat for a long period of time, which might be sufficient to kill all pathogens. One of the limitations of our study is that the control group was not from people in the general population of the four villages. However, in this approach, the results would perhaps be influenced by the effects of other social events apart from the funeral. Since all the cases in our study attended the funeral, therefore, we think that taking the controls from the same event might avoid other influences. Another limitation of this study is that controls were not tested for asymptomatic or subclinical hepatitis A by liver biochemistry and serology.

A sample of nipa palm wine that was consumed during the ceremony was not available for investigating the presence of $\mathrm{HAV}$, as the event had occurred much earlier. The average $\mathrm{pH}$ of the nipa palm wine tested was 6.82, at which HAV can survive and multiply. HAV is stable in alcohol, as disinfection with alcohol has been found to be ineffective against HAV on surfaces. ${ }^{16}$ Our investigation showed that the probable contamination of nipa palm wine with HAV has occurred at any point of its processing, as sap collection and fermentation site were unhygienic. However, in the present study, HAV contamination most likely occurred during the dilution of nipa palm wine with river water. We found undocumented immigrant houses on the riverside, where sanitary facilities were rudimentary and may have acted as a source of river water contamination.

Hepatitis A virus has been shown to have a single conserved antigenic neutralization site; therefore, all isolates from different parts of the world belong to a single serotype. ${ }^{17}$ However, the VP1 region of HAV contains variable amino acids and is used for molecular detection. ${ }^{18}$ Variable regions within the capsid proteins, including the VP3/VP1 and VP1/P2A junctions, have been used to compare sequences of isolates obtained from several countries. ${ }^{18}$ This indicates some degree of genomic diversity, resulting in the classification of HAV into six genotypes (I-VI). ${ }^{17}$ Genotypes I-III have been associated with infections in humans and are further divided into two subgenotypes (A and B). Genotypes and subgenotypes are often associated with different geographic distributions. ${ }^{17}$ In general, genotype $\mathrm{I}$ is the most prevalent, with subtype IA being more common than IB. Viral genotypic profiles are required to identify foodborne outbreaks to implement preventative measures and recognize transmission routes. The results of the present study demonstrated that all isolates in this outbreak belonged to subgenotype IA. The HAVs identified in our study displayed a high degree of genetic conservation, and clustered together in the phylogenetic tree.

The identification of a single cluster of HAV in the present situation indicates that this was a single-source outbreak. Phylogenetically, our strains were related to the HAV from a patient identified in Fukuoka, Japan, in 2004 and to outbreak strains reported in 2015 among men who had sex with men in Taiwan. Our strains were also phylogenetically related to an environmental strain from the Paranaque River in metropolitan area of Manila, the Philippines. As this strain is circulating in a wide area of Southeast Asia, we believe that this is an emerging strain of HAV. Therefore, further investigation is needed for the assessment of the geographical extent of HAV.

\section{CONCLUSION}

An outbreak of hepatitis A by an HAV of genotype IA was confirmed to be caused by consuming nipa palm wine at a funeral ceremony. The wine was homemade and mixed with river water during its preparation, which might have contained 
the virus. The strain is possibly an emerging HAV strain in Asia. We managed to identify the nipa palm wine maker and advised him on hygienic preparation of palm wine. We recommend that health education programs and strict monitoring as well as continuous close surveillance of traditional palm wine production should be implemented by the local authority.

Received January 11, 2021. Accepted for publication May 6, 2021.

Published online July 12, 2021.

Note: Supplemental figure appears at www.ajtmh.org.

Acknowledgments: We would like to thank the Director General of Health Malaysia for his permission to publish the findings and the Director of the Sabah State Health Department for her support. We also would like to thank all the staffs of Sabah State Health Department for their contributions to this study.

Financial support: The investigation was undertaken within the Ministry of Health activities and supported by Launching Grant for Centre of Excellence from Universiti Malaysia Sabah (Grant Number: AM 18006).

Authors' addresses: Muhammad Jikal, M. Hafiz Mukhsam, Ismail Ali, and Esther Bernad, Sabah State Health Department, Ministry of Health, Kota Kinabalu, Sabah, Malaysia, E-mails: drmj70@gmail. com, hafizz_mhm@yahoo.com, drismailali@moh.gov.my, and oharaeb@gmail.com. Daisuke Mori and Kamruddin Ahmed, Department of Pathobiology and Medical Diagnostics, Faculty of Medicine and Health Sciences, Universiti Malaysia Sabah, Kota Kinabalu, Sabah, Malaysia, E-mails: daisuke.mori.0128@gmail.com and ahmed@ums.edu.my. Ahmad Faudzi Yusoff, Institute of Medical Research, Ministry of Health, Jalan Pahang, Kuala Lumpur, Wilayah Persekutuan Kuala Lumpur, Malaysia, E-mail: faudziyusoff@gmail. com. Sarawasthi Bina Rai, Aimst University, Bedong, Kedah, Malaysia, E-mail: binarai@yahoo.com. Liza Abd Latif, Cynthia C. E. Bailon, and Lim Guan Lee, Beaufort Health Office, Ministry of Health, Pekan Beaufort, Beaufort, Sabah, Malaysia, E-mails: liza_emily@ yahoo.com, cceb1092@gmail.com, and Iglee@moh.gov.my. Alfred A. S. Perianayagam and Vielerian Fredolin, Papar Health Office, Ministry of Health, Bandar Papar, Papar, Sabah, Malaysia, E-mails: draldrin.constantine9@gmail.com and iyanvell23@gmail.com.

\section{REFERENCES}

1. World Health Organization, 2012. WHO position paper on hepatitis A vaccine. Wkly Epidemiol Rec 87: 15.

2. Lemon SM, Ott JJ, Van Damme P, Shouval D, 2017. Type A viral hepatitis: a summary and update on the molecular virology, epidemiology, pathogenesis and prevention. $\mathrm{J} \mathrm{Hepatol}$ 68: 167-184.

3. Raihan R, 2016. Hepatitis in Malaysia: past, present, and future. Euroasian J Hepatogastroenterol 6: 52-55.
4. WHO, 2018. Malaysia: Highlights Based on Country Reported GLAAS 2016/2017 Data. Available at: https://www.who.int/ water_sanitation_health/monitoring/investments/countryhighlights-2017/malaysia-glaas2017-country-highlight2018 1115.pdf?ua=1. Accessed April 11, 2021.

5. Murphy A, Rajahram GS, Jilip J, Maluda M, William T, Hu W, Reid S, Devine GJ, Frentiu FD, 2020. Incidence and epidemiological features of dengue in Sabah, Malaysia. PLoS Negl Trop Dis 14: e0007504

6. Malaysia $\mathrm{MoH}, 2006$. Garis Panduan Umum Pengurusan Wabak Penyakit - Penyakit Bawaan Makanan dan Air di Malaysia Jlid 1 - FWBD UMU GP 001 (pindaan_2006). Available at: https:// www.moh.gov.my/index.php/pages/view/118? $\mathrm{mid}=48$. Accessed April 12, 2021.

7. Tominaga A et al., 2012. Hepatitis A outbreak associated with a revolving sushi bar in Chiba, Japan: application of molecular epidemiology. Hepatol Res 42: 828-834.

8. Barzaga BN, 2000. Hepatitis A shifting epidemiology in southeast Asia and China. Vaccine 18: S61-S64.

9. Wang Z, Chen $Y, X i e ~ S, ~ L v ~ H, ~ 2016$. Changing epidemiological characteristics of hepatitis $A$ in Zhejiang Province, China: increased susceptibility in adults. PLoS One 11: e0153804.

10. Smith CR, Kershaw T, Johnson K, Meghnath K, 2019. An outbreak of hepatitis A in Canada: the use of a control bank to conduct a case-control study. Epidemiol Infect 147: e300.

11. Yusoff FA, Rahman RA, May LH, Budart SB, Sulaiman LH, 2015. Investigation of hepatitis A outbreak in district of Manjung, Perak, Malaysia, October 2012. Western Pac Surveill Response J 6: 27-31.

12. Sowmyanarayanan TV, Mukhopadhya A, Gladstone BP, Sarkar R, Kang G, 2008. Investigation of a hepatitis A outbreak in children in an urban slum in Vellore, Tamil Nadu, using geographic information systems. Indian J Med Res 128: 32-37.

13. Desenclos JA, Klontz KC, Wilder MH, Gunn RA, 1992. The protective effect of alcohol on the occurrence of epidemic oyster-borne hepatitis A. Epidemiol 3: 371-374.

14. Shoesmith WD, Oo Tha N, Naing KS, Abbas RB, Abdullah AF, 2016. Unrecorded alcohol and alcohol-related harm in rural Sabah, Malaysia: a socio-economically deprived region with expensive beer and cheap local spirits. Alcohol Alcohol 51: 741-746.

15. Lewallen $S$, Courtright $P, 1998$. Epidemiology in practice: casecontrol studies. Community Eye Health 11: 57-58.

16. Mbithi JN, Springthorpe VS, Sattar SA, 1990. Chemical disinfection of hepatitis A virus on environmental surfaces. Appl Environ Microbiol 56: 3601-3604.

17. Nasheri N, Vester A, Petronella N, 2019. Foodborne viral outbreaks associated with frozen produce. Epidemiol Infect 147: e291.

18. Setyowati D, Mubawadi T, Mirasa YA, Purwanto D, Amin M, Utsumi T, Soetjipto S, Juniastuti J, Lusida MI, 2020. Molecular epidemiology of hepatitis A outbreaks in two districts in Indonesia in 2018: same subtype, but different strains. Biomed Rep 12: $51-58$. 